\title{
Multiple primary malignant neoplasms: A case report and literature review
}

\author{
LINWEI ZHANG ${ }^{*}$, LINGXIN FENG ${ }^{*}$, HUI CONG, ZHUANG YU, HAOCHENG WANG, YA DONG and JING WANG \\ Department of Oncology, The Affiliated Hospital of Qingdao University, Qingdao, Shandong 266000, P.R. China
}

Received March 5, 2019; Accepted July 9, 2019

DOI: $10.3892 /$ ol.2019.10779

\begin{abstract}
Until recently, few cases of three or more malignant tumors in one patient have been reported. Owing to the high incidence rate of these tumors, the improvement in cancer diagnosis and treatment, and the extension of patient survival time, the incidence of reported multiple primary malignant neoplasms has gradually increased. The present study reported the case of a 57-year-old man with non-small cell lung cancer combined with B-Raf proto-oncogene serine/threonine kinase V600E mutation, gastrointestinal stromal tumors and lumbar vertebral malignant mucinous sarcoma. The pathogenesis, diagnosis and treatment of these three malignancies are discussed and previous studies are also reviewed. The aim of the study was to analyze the genetic mutations associated with multiple primary malignant tumors and to discuss whether those mutations with unknown functional significance could be used as therapeutic indicators. This case report will serve as a reference for future treatment of such patients.
\end{abstract}

\section{Introduction}

The incidence rate for multiple primary malignant neoplasms (MPMNs) is estimated to be between 0.73 and $11.7 \%$ (1). MPMNs are defined as two or more unassociated primary malignant tumors that occur in the body synchronously or metachronously (2). Each tumor originates from different tissues and organs, presents as a distinct pathological type and excludes lesions that are secondary or metastatic to other tumors (3). MPMNs can be divided into two categories (3): i) Synchronous, defined as malignancies that occur within 6 months of the diagnosis of a previous malignant neoplasm;

Correspondence to: Professor Jing Wang, Department of Oncology, The Affiliated Hospital of Qingdao University, 7 Jiaxing Road, Shibei, Qingdao, Shandong 266000, P.R. China

E-mail: wangstella5@163.com

*Contributed equally

Key words: multiple primary malignant neoplasms, non-small cell lung cancer, BRAF V600E mutation, gastrointestinal stromal tumor, malignant fibromyxoid sarcoma, imatinib, dabrafenib, trametinib, PD-L1 and ii) metachronous, defined as malignancies that occur $>6$ months apart. In a recent study, the risks of developing second primary cancers were higher in cancer survivors compared with the general population with a $3.8 \%$ higher incidence of metachronous second primary cancers within a median follow-up time of 2.5 years; furthermore, the estimated 10-year cumulative risk of second primary cancers for patients who were firstly diagnosed with cancer aged between 60 and 69 was as high as $13 \%$ (4). Compared with a single primary tumor, MPMNs have increased malignant behavior and a worse prognosis (5).

\section{Case report}

A 57-year-old male was admitted to the Chinese People's Liberation Army 401 Hospital (Qingdao, China) with pain in the left upper abdomen in August 1996. Enhanced computed tomography (CT) scans revealed a $10 \times 9.5 \times 12-\mathrm{cm}$ soft-tissue mass, with heterogeneous density on the greater curvature. A gastric leiomyosarcoma was suspected and thus, the patient underwent resection of the gastric lesion and partial transverse colon on August 21, 1996. Postoperative pathological diagnosis confirmed gastric leiomyosarcoma with low-grade malignancy. After 11 years, in April 2007, an abdominal CT scan was performed due to abdominal pain, which demonstrated a low-density, round, soft-tissue mass located between the liver and stomach. Considering it a recurrent tumor, surgeons performed a resection of the gastric lesion and a left hepatic lobectomy on April 29, 2007. Postoperative pathological diagnosis confirmed it as a low-grade malignant gastrointestinal stromal tumor (GIST). The immunohistochemical results are presented in Fig. 1. In April 2013, the annual routine re-examination revealed disease recurrence again; two large cystic lesions in the abdomen were discovered by a CT scan, one of which was found between the caudate lobe of the liver and the right side of the fundus of the stomach, and the other of which was found on the retroperitoneum. Considering the previous diagnosis of a GIST, the surgeon did not preform a biopsy again before surgery. The patient underwent a total gastrectomy, as well as stern resection of the pancreas, splenectomy, left adrenalectomy and Roux-en-Y esophagojejunostomy. Following this major surgery, the postoperative reactions of the patient included weight loss and impaired digestive function. The postoperative pathology confirmed GIST and the immunohistochemistry results of the tumor are presented in Fig. 2. The 

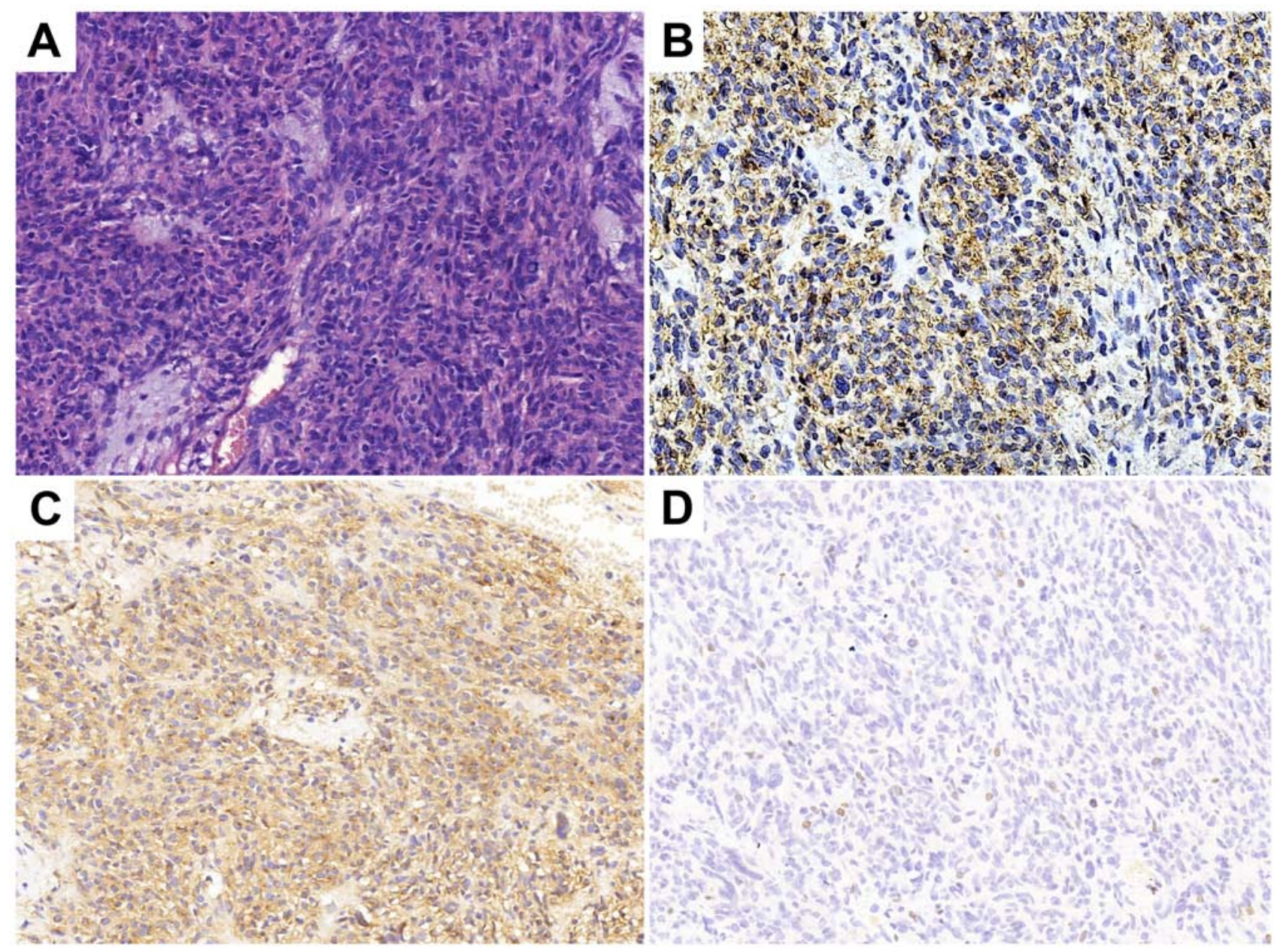

Figure 1. Histopathological analysis following gastric lesion resection and left hepatic lobectomy in 2007. (A) Hematoxylin and eosin staining indicating the tumor as a low-grade malignant gastrointestinal stromal tumor. Tumor cells were (B) CD34 (++), (C) CD117 (+++) and (D) Ki-67 (+, <10\%). Magnification, x 200 .

patient was prescribed daily oral imatinib (400 mg) from May 2013. During this period, diarrhea and edema occurred, which spontaneously resolved after 2 months and thus, the dosage of imatinib was not decreased.

The patient was referred to the Qilu Hospital of Shandong University in November 2015 due to pain on the left side of the back. As shown in Fig. 3, there was an irregular lesion measuring $4.2 \times 5.7 \times 6.7 \mathrm{~cm}$ between the left transverse process of the 5th lumbar vertebrae (L5) and the sacroiliac joint, partly involving the sacrum and left iliac bone. The CT scan showed heterogeneous enhancement. The patient underwent surgery to remove the lumbar soft tissue tumors on January 11,2016 , and the postoperative pathology indicated malignant ossifying myxofibrosarcoma (MFS) with infiltrating growth and satellite nodules besides the main body of the tumor. The immunohistochemistry results were as follows: Smooth muscle actin positivity, CD34 positivity, cyclin-dependent kinase 4 (CDK-4) positivity, p63 negativity, S-100 negativity, glial fibrillary acidic protein negativity, epithelial membrane antigen (EMA) negativity, pan-cytokeratin (CKpan) negativity, vimentin (foci) positivity and Ki-67 positivity (1-2\%) (Fig. 4). Annual re-examination of $\mathrm{CT}$ and magnetic resonance imaging scans after the surgery showed no obvious changes in L5.
On March 6, 2018, a routine CT scan revealed two hypoechoic nodules in the right liver with a bull's-eye configuration. Multiple enlarged lymph nodes were found on the right anterior cervical region through physical examination. A cervical lymph node dissection biopsy was performed, and pathological examination revealed metastatic low-differentiated adenocarcinoma of the right cervical lymph nodes. Immunohistochemistry results showed positivity for EMA, CKpan, cytokeratin (CK) 7, CK19, carcinoembryonic antigen, transcription termination factor 1 (TTF-1) and S-100 (Fig. 5), supporting the hypothesis that the tumor was derived from the lung, and it was classified as adenocarcinoma. When these findings were combined with the results from a positron emission tomography (PET)-CT scan (Fig. 6), pulmonary adenocarcinoma located on the inferior lobe of the right lung and liver metastases were diagnosed. Clinical Tumor-Node-Metastasis stage was shown to be T1bN3Mx. Next-generation sequencing technology (NGS) was used on tissues and revealed positivity for the B-Raf proto-oncogene serine/threonine kinase (BRAF) V600E mutation (Table I). NGS analyses were performed using a long-range PCR-based target enrichment method (LR-PCR-based NGS) (6). Considering the poor physique and intolerance of the patient to chemotherapy, targeted therapy was recommended. The patient was recommended dabrafenib 

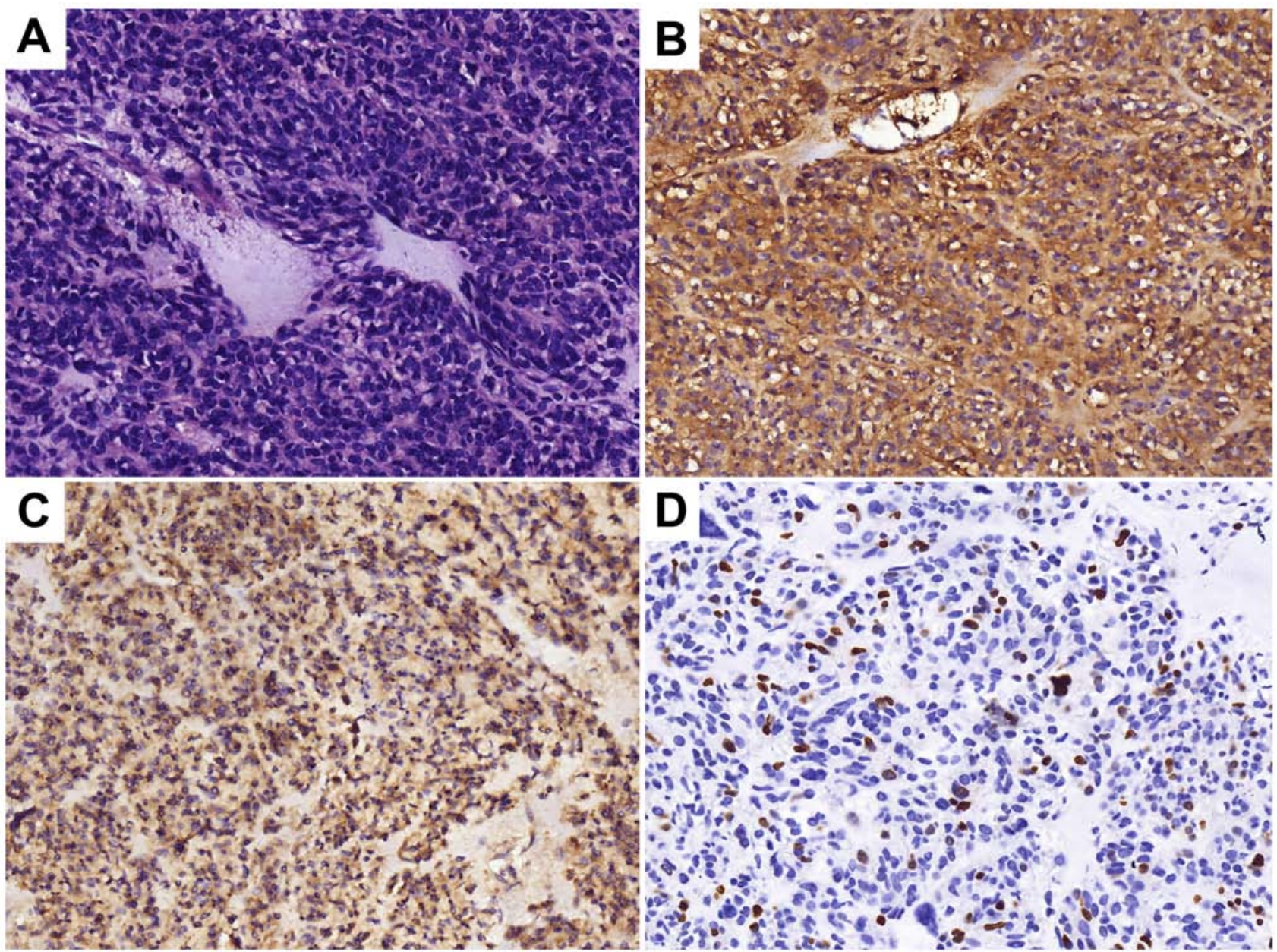

$D^{10}=0$

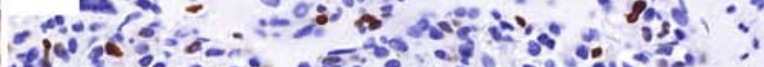

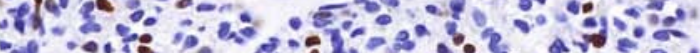

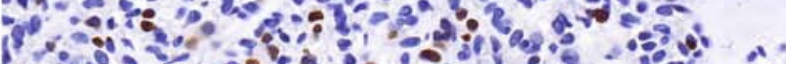

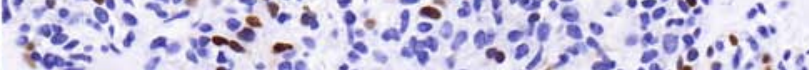

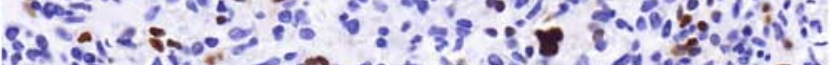
82.

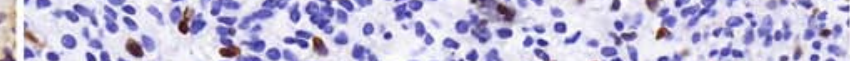

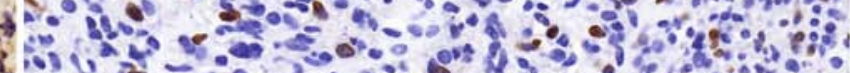

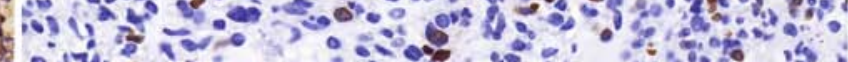

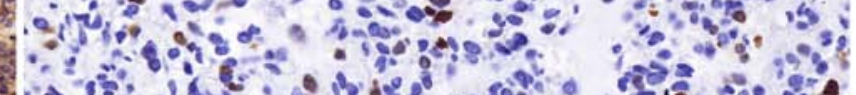

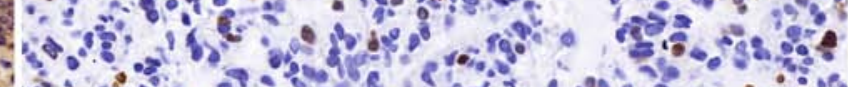
a

Figure 2. Histopathological analysis following total gastrectomy, stern resection of the pancreas, splenectomy, left adrenalectomy and Roux-en-Y esophagojejunostomy in 2013. (A) Hematoxylin and eosin staining indicating a malignant gastrointestinal stromal tumor. Tumor cells were (B) CD34(+), (C) CD117(+/-) and (D) Ki-67(+, 20\%). Magnification, x200.
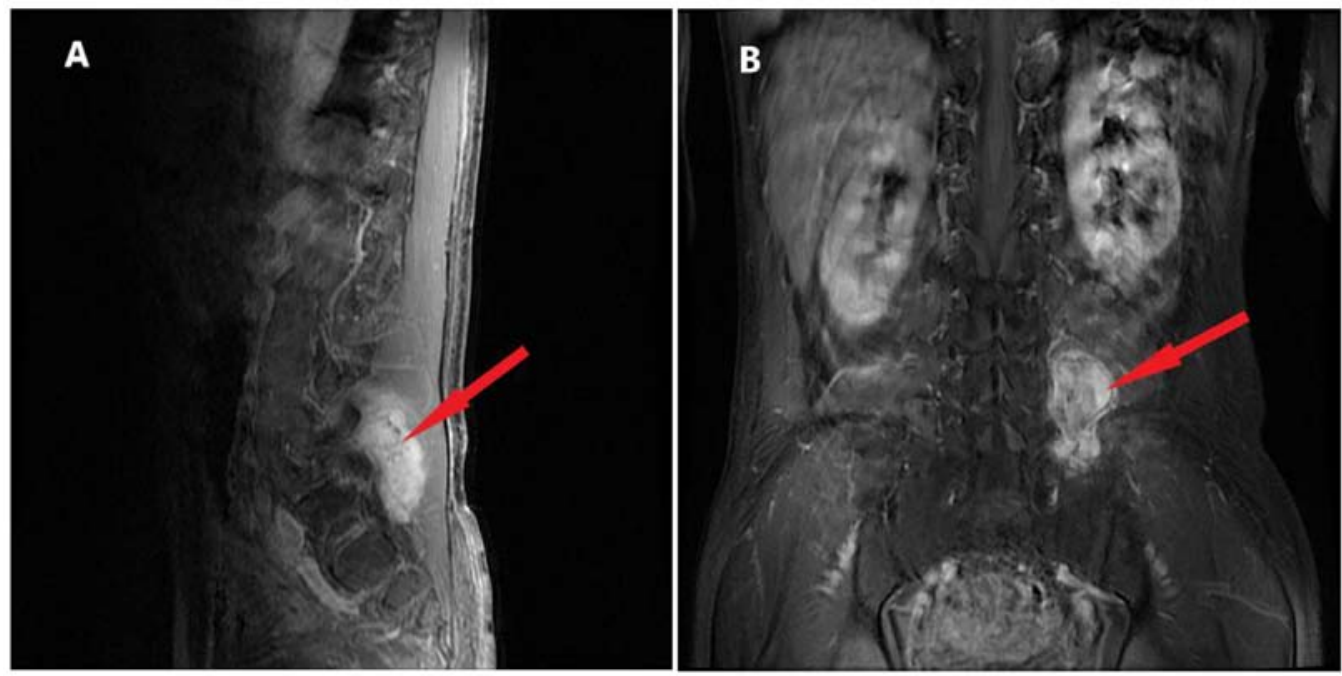

Figure 3. Magnetic resonance imaging of the L5 prior to the operation in 2015. (A) Sagittal and (B) coronal position showing the irregular lesion (red arrow) of $4.2 \times 5.7 \times 6.7 \mathrm{~cm}$ between the left transverse process of the $\mathrm{L} 5$ and sacroiliac joint, partly involving the sacrum and left iliac bone. L5, 5th lumbar vertebra.

(300 $\mathrm{mg} /$ day) and trametinib (2 $\mathrm{mg} /$ day) as therapy from April 2018, and no obvious adverse reactions were noted. On June 8, 2018, and August 3, 2018, re-examination of the chest by a CT scan (Fig. 7) revealed significant reduction in the size and number of the nodule in the inferior lobe of the right lung, the multiple pulmonary nodules and the lymph nodes 

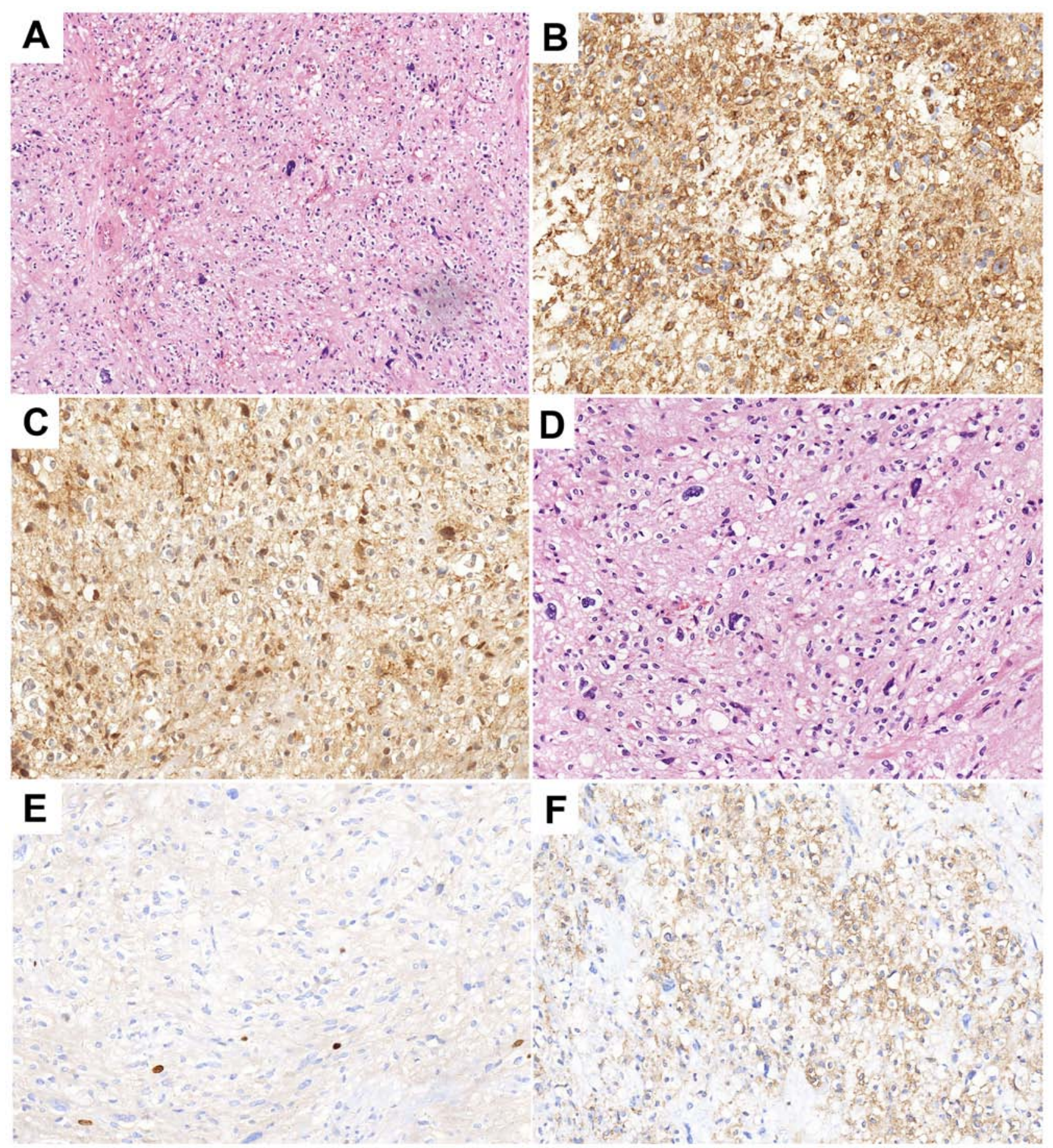

Figure 4. Histopathological analysis results following the surgery in 2016. (A and B) Hematoxylin and eosin staining indicating a malignant ossifying fibromyxoid sarcoma with infiltrating growth and satellite nodules besides the main body of the tumor. Tumor cells were (C) CD34(+), (D) Ki67(+, 1-2\%), (E) cyclin-dependent kinase $4(+)$, and (F) smooth muscle actin (+). Magnification, (A) x100 and (B and C).

in the mediastinum. According to the Response Evaluation Criteria In Solid Tumors (RECIST version 1.1) (7), the evaluation result indicated stable disease (SD). On October 242018 , re-examination of the upper abdomen by a CT scan (Fig. 8) revealed larger hypoechoic nodules in the right liver compared with those observed on the previous CT scan. This demonstrated disease progression. To evaluate the properties of the intrahepatic lesions, a CT-guided percutaneous liver biopsy was performed on October 29, 2018. Pathological examination revealed invasion of malignant tumor cells in liver tissue.
Furthermore, immunohistochemistry results showed positivity for CKpan, CK7, p40, Napsin A part, TTF-1, and negativity for CD117, Dog-1, CD34, hepatocyte antigen, arginase-1 and programmed death ligand 1 (PD-L1; 70\%), supporting the hypothesis that the intrahepatic lesions were metastases from lung cancer. Following this, two cycles of chemotherapy, pemetrexed (700 mg, on day 2 every 3 weeks) combined with bevacizumab (400 mg, on day 1 every 3 weeks), were performed. The evaluation of the effect of these cycles of chemotherapy indicated SD according to RECIST version 1.1. 

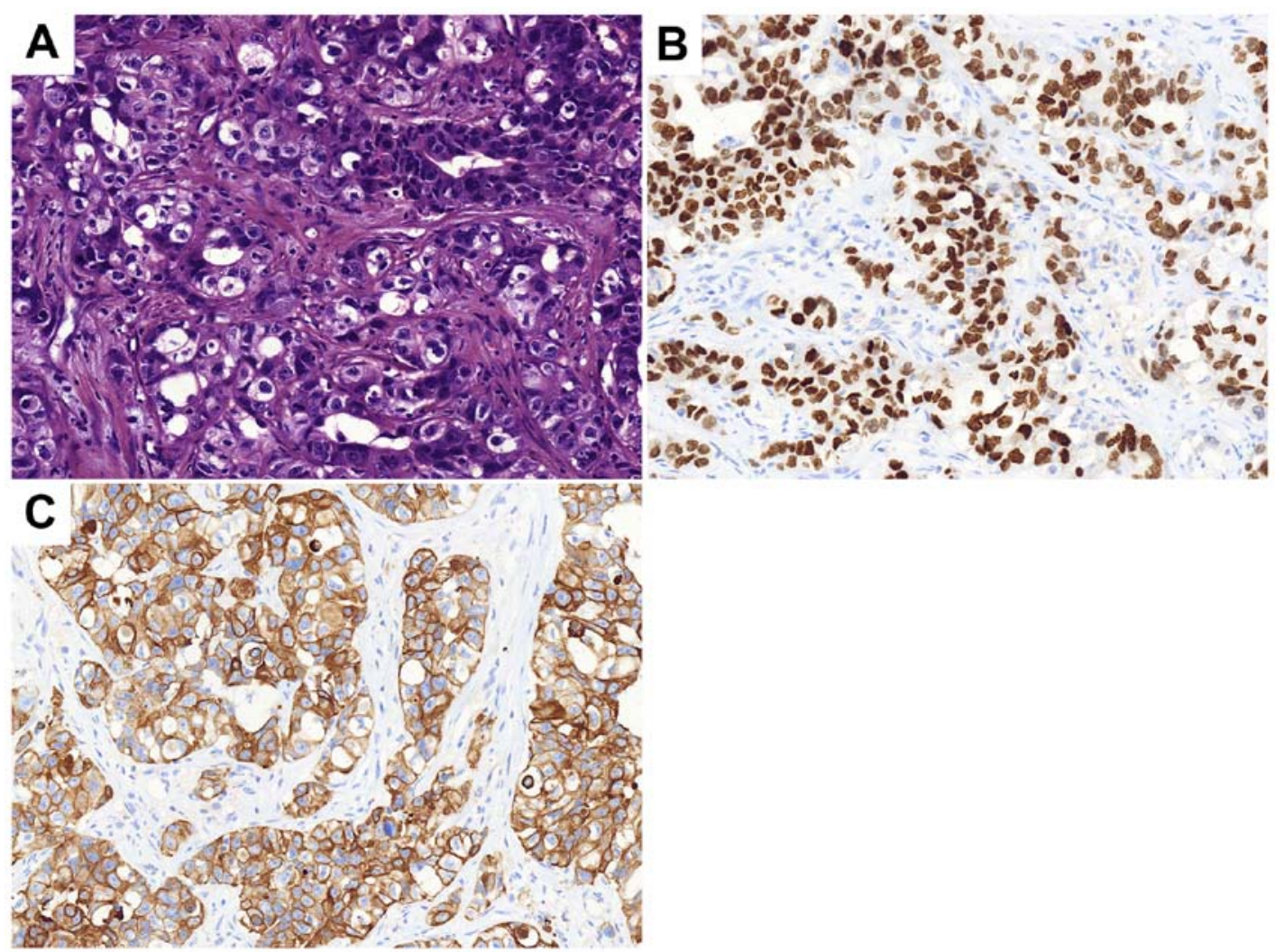

Figure 5. Histopathological analysis results following cervical lymph node dissection and biopsy in 2018. (A) Hematoxylin and eosin staining indicating metastatic low-differentiated adenocarcinoma of the third and fourth group of the right cervical lymph nodes. Tumor cells were (B) transcription termination factor $1(+)$ and (C) cytokeratin 7 (+). Magnification, x200.
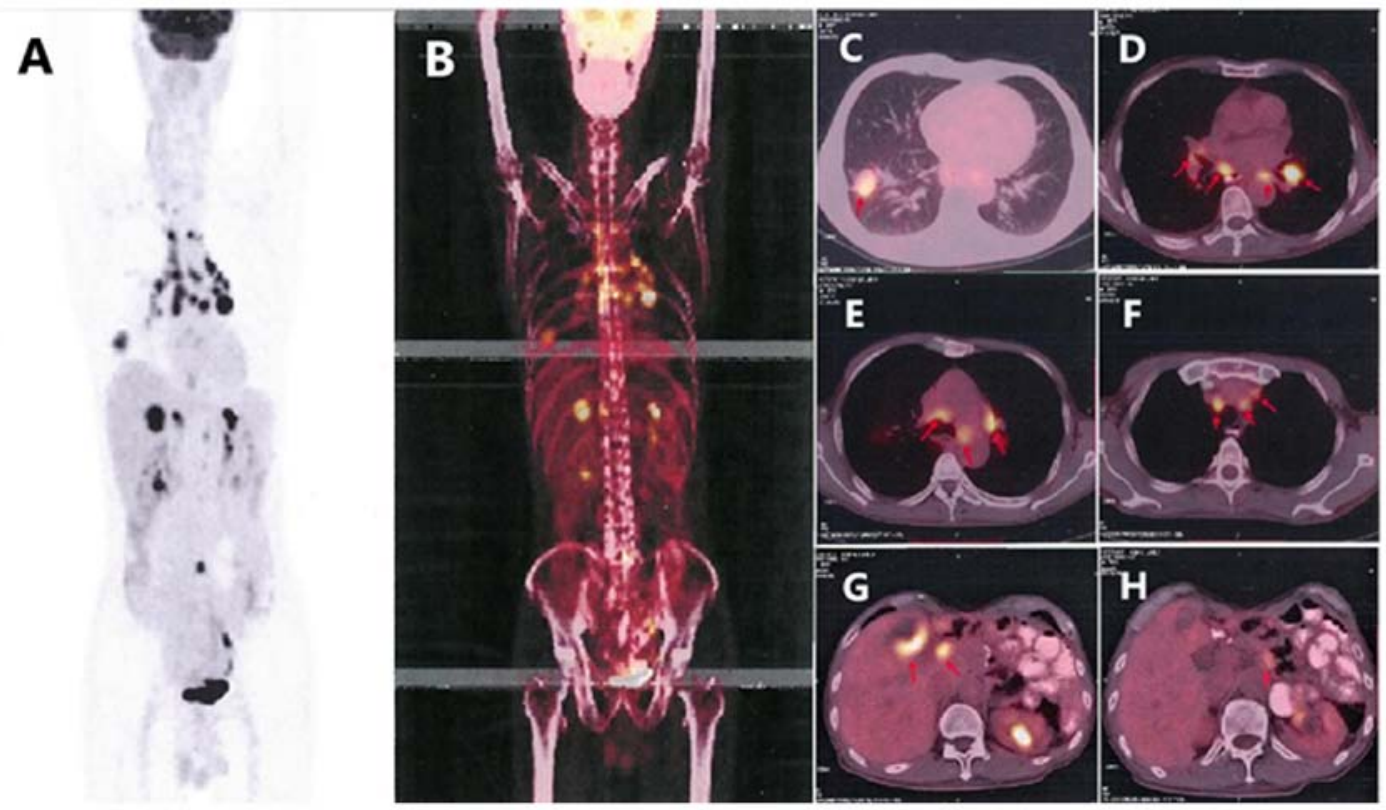

Figure 6. Positron emission tomography-contrast enhanced scan. (A and B) The scan of the whole body. Metabolic activity was observed in (C) the right lower lobe (red arrow), (D-F) the mediastinal lymph nodes (red arrow) and (G) the liver (red arrow). The tumor is located on (C) the inferior lobe of the right lung (red arrow), and (G) liver metastases (red arrow) was considered to be pulmonary adenocarcinoma. (H) The lymph nodes (red arrow) adjacent to the abdominal aorta

\section{Discussion}

MPMNs are defined as the occurrence of two or more primary malignant tumors, each occurring at a different site and not representing extension, recurrence or metastasis (8). According to Warren and Gates (9), if the interval between occurrences is $<6$ months, the diagnosis is considered to be of synchronous MPMN, and if $>6$ months, the diagnosis is considered to be 

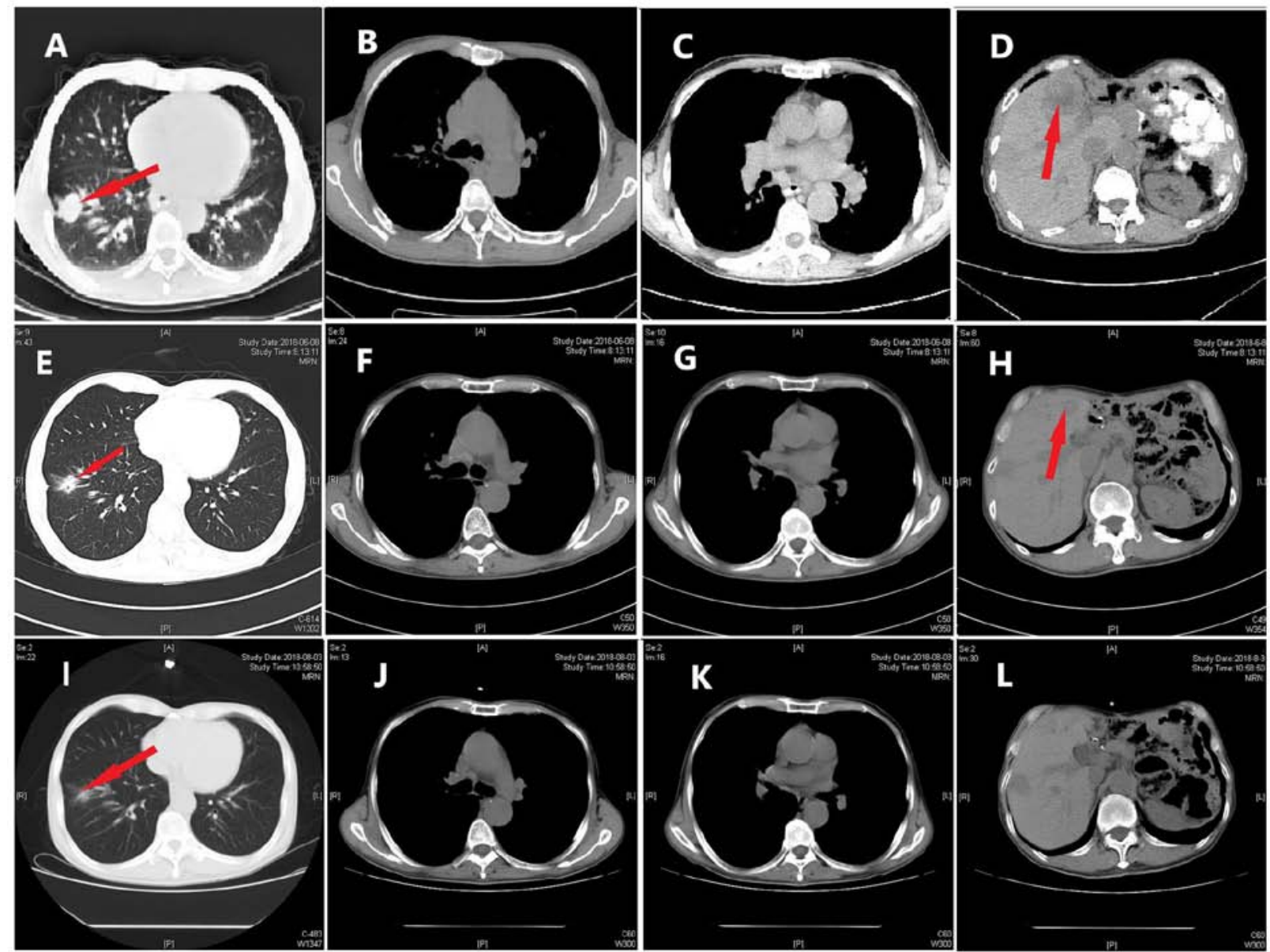

Figure 7. CT scans of foci in the lung and liver. CT scans (A-D) prior to treatment, and after (E-H) 2 and (I-L) 4 months of oral dabrafenib and trametinib treatment. CT, computed tomography. Red arrows indicate (A, E and I) the lesion in right lung and (D and H) the lesion in the liver.

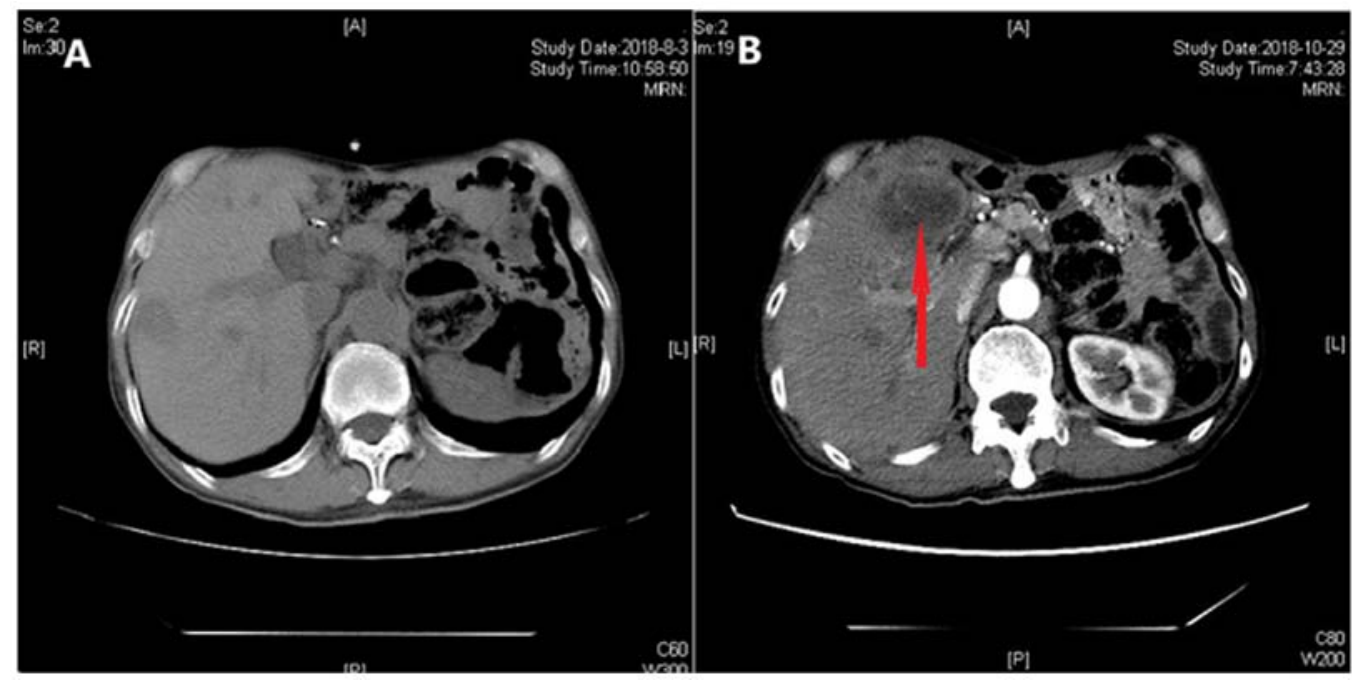

Figure 8. CT scans of focus in the liver. CT scans (A) 4 and (B) 6 months after oral dabrafenib and trametinib treatment. The red arrow indicates the lesion in the liver. CT, computed tomography.

a metachronous MPMN (10). The patient in the present case report conformed to the diagnosis of metachronous MPMN for the following reasons: Firstly, the patient suffered from three tumors, namely a GIST, malignant fibrous mucinous sarcoma of the lumbar spine and lung adenocarcinoma. Secondly, the onset time of these three malignant tumors exceeded the cut-off time of 6 months. Finally, the pathological types of these three malignant tumors were different, and showed no recurrence or metastases. The pathogenic factors of MPMNs are unclear, but may be associated with the following factors: i) Endogenous 
Table I. Mutation frequency detected by next-generation sequencing technology.

\begin{tabular}{|c|c|c|c|c|}
\hline Gene & Base mutation & Amino acid mutation & Gene subregion & Mutation frequency, $\%$ \\
\hline BRAF & c. $1799 \mathrm{~T}>\mathrm{A}$ & p.V600E & EX15 & 27.69 \\
\hline TP53 & c. $811 \mathrm{G}>\mathrm{A}$ & p.R237H & EX8 & 24.57 \\
\hline EGFR & c. $728 \mathrm{C}>\mathrm{G}$ & p.P234R & EX6 & 25.30 \\
\hline NTRK3 & c. $1685 \mathrm{C}>\mathrm{T}$ & p.P562L & EX15 & 21.64 \\
\hline BTK & c. $286 \mathrm{G}>\mathrm{A}$ & p.E96K & EX4 & 20.83 \\
\hline PDGFRA & c. $509 \mathrm{C}>\mathrm{T}$ & p.A170V & $\mathrm{EX} 3$ & 9.12 \\
\hline
\end{tabular}

EGFR, epidermal growth factor receptor; NTRK3, NT-3 growth factor receptor; BTK, Bruton tyrosine kinase; PDGFRA, platelet-derived growth factor receptor $\alpha$; TP53, tumor protein $\mathrm{p} 53$; BRAF, B-Raf proto-oncogene serine/threonine kinase.

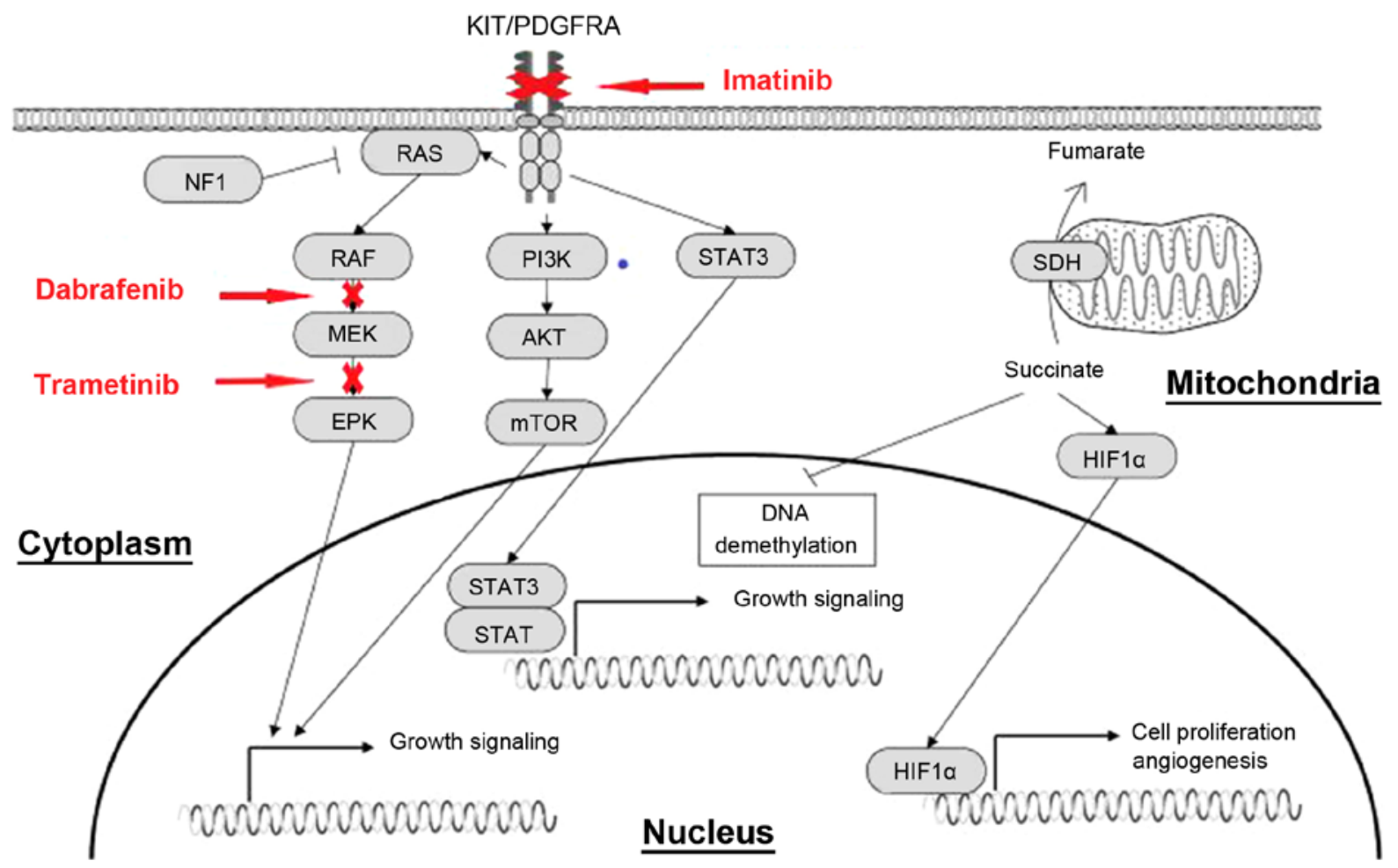

Figure 9. Effect of imatinib, dabarafenib and trametinib on downstream signaling pathways. Activating mutations of KIT and PDGFRA genes permit ligand-independent phosphorylation of the receptor tyrosine kinases, allowing the receptor-initiated signal and causing activation of the downstream effectors, such as PI3K/AKT, Ras/MAPK and JAK/STAT. PDGFRA, platelet-derived growth factor receptor $\alpha$; JAK, Janus kinase; MAPK, mitogen-activated protein kinase; PI3K, phophoinositide-3 kinase; mTOR, mechanistic target of rapamycin; STAT, signal transducer and activator of transcription; c-Kit, KIT proto-oncogene receptor tyrosine kinase; HIF1 $\alpha$, hypoxia inducible factor- $1 \alpha$; SDH, succinate dehydrogenase; ERK, extracellular regulated protein kinases.

factors such as abnormal development of the embryo, immunity-associated diseases and endocrine diseases affecting carcinogen sensitivity; ii) environmental exposure, including long-term effects of radiation and industrial pollution, and lifestyle; iii) genetic determinants; and iv) iatrogenic effects, particularly radiation therapy and drug therapy (11). There are no currently available standard guidelines for MPMN treatment. Malignancy type, disease stage and the overall health of the patient are each considered (12), and each patient has multidisciplinary individualized treatment.

The patient evaluated in the present case report successively suffered from GIST, MFS and NSCLC. The patient had no immune-associated diseases, no history of exposure to toxic and harmful substances, and the family history revealed that the patient's brother died of lung cancer several years ago. Similarities in gene abnormalities, lifestyle and living environment to the brother may have been associated with the disease status of the patient. Following the diagnosis of NSCLC in 2018, NGS was performed on the tumor tissue, which revealed mutations in tumor protein p53, epidermal growth factor receptor (EGFR), NT-3 growth factor receptor (NTRK3), Bruton tyrosine kinase (BTK), and platelet-derived growth factor receptor $\alpha$ (PDGFRA) (Table I). BRAF V600E mutation has clinical significance, and is associated with the occurrence of multiple tumors, including NSCLC and a few non-KIT proto-oncogene receptor tyrosine kinase KIT/PDGFRA-mutated GISTs (13). 
The mutations in EGFR, NTRK3, BTK and PDGFRA genes are uncommon missense mutations that have ambiguous significance, nevertheless, they are not considered irrelevant to the occurrence of multiple tumors in the patient mentioned in the present case report. Since 2013, the patient was taking oral imatinib for the GIST. Thus, long-term application of targeted drug therapy may also increase the incidence of secondary malignant tumors. In 2002, Surveillance Epidemiology and End Results data indicated that $\sim 16 \%$ of all new cancer cases were secondary or higher-order primary cancer in the United States (12).

The BRAF gene is mutated in $1-5 \%$ of NSCLC cases and most of these mutations occur in adenocarcinoma (14). BRAF is a member of the RAF family of serine/threonine kinases that mediate signal transduction between RAS and mitogen-activated protein kinase (MAPK) signaling pathways (15). Once the mutation is activated, the downstream mitogen-actiavted protein kinase (MEK)-extracellular regulated protein kinase signaling pathway is activated continuously, resulting in the excessive proliferation and survival of tumor cells (Fig. 9) (16). The V600E mutation accounts for $\sim 50 \%$ of BRAF mutations (14). In a multicenter, single arm, non-randomized phase II study (BRF113928; ClinicalTrials. gov identifier, NCT01336634) (17), the efficacy of dabrafenib as a monotherapy was compared with that of dabrafenib administered in combination with trametinib. The primary endpoint included the objective response rate (ORR), disease control rate (DCR), median progression-free survival (PFS) time and overall survival (OS) time of 84 patients treated with oral dabrafenib. Evaluation of these primary endpoints in patients treated with dabrafenib alone revealed the following: ORR, 33\%; DCR, 58\%; PFS time, 5.5 months; and OS time, 12.7 months. The ORR, DCR and median PFS time of the patients who were treated with dabrafenib and trametinib were $63,79 \%$ and 9.7 months, respectively, and $65 \%$ of the patients achieved a PFS time of $>6$ months (17). Thus, the combination of MEK inhibitors with BRAF inhibitors may demonstrate improved results compared with BRAF inhibitors alone (18). The patient in the present case report was diagnosed with NSCLC harboring a BRAF V600E mutation. The Food and Drug Administration regulations approved dabrafenib and trametinib in combination for treatment of metastatic NSCLC with a BRAF V600E mutation on June 22, 2017 (19), therefore combined dabrafenib and trametinib treatment was administered. At 6 months after the treatment, the lesions in the liver had progressed, which suggested drug resistance. Kim et al (20) recently reported that the expression of PD-L1 was changed in $28 \%$ of patients following EGFR-tyrosine kinase inhibitor (TKI) therapy. Su et al (21) performed a retrospective analysis, which revealed high PD-L1 expression to be a predictor of a poor response to EGFR-TKI treatment among patients with EGFR-mutated NSCLC. Thus, resistance to the BRAF inhibitor may be associated with the high PD-L1 expression in the patient described in the present case report. A previous study has demonstrated that, in melanoma models, cells resistant to BRAF inhibitors exhibited increased MAPK signaling and PD-L1 expression (22). Moreover, increased expression of markers of T-cell exhaustion and PD-L1 were detected in patients with melanoma following treatment with a BRAF inhibitor. Immunotherapy plays an important role in advanced NSCLC patients with high expression of PD-L1. In a phase II study, the combination of pembrolizumab with carboplatin and pemetrexed in chemotherapy-naïve, advanced non-squamous NSCLCs was evaluated (23). The pembrolizumab combined with chemotherapy group achieved an ORR of $55 \%$ in comparison to $29 \%$ in the chemotherapy alone group (23). The incidence of grade 3 or worse treatment-related adverse events was similar between the two groups. PFS time was also significantly longer in patients treated with pembrolizumab in combination with chemotherapy compared with that in patients who received chemotherapy alone (23).

GISTs are known to be the most common type of mesenchymal neoplasm of the gastrointestinal tract. From a previous study, the worldwide incidence and prevalence rates were estimated to be between 1 and 1.5 per 100,000 per year and 13 per 100,000 individuals, respectively in 2005 (24). Peixoto et al (25) demonstrated that the stomach was the most frequently involved organ with regard to GISTs, accounting for $64.1 \%$ of all cases, and that the lesions were distributed in the fundus (15.5\%), body (61.9\%) and antrum (22.6\%) (25). Other organs that were involved included the small bowel $(29.8 \%)$, duodenum (30.8\%), jejunum (30.8\%) and ileum $(38.5 \%)$. Occasionally, the omentum, mesentery and peritoneum may also be affected. GIST metastases often involved the liver and could present as a primary disease or as recurrence following surgery (26).

A GIST is associated with a mutation in the KIT gene ( $\sim 80 \%$ of cases) or the PDGFRA gene, coding for type III receptor tyrosine kinases (27). Mutations in the PDGFRA gene can be detected in $\sim 10 \%$ of GISTs without a KIT gene mutation. The landscape of mutations in PDGFRA mostly occur in exon 18, accounting for $>90 \%$ of all PDGFRA mutations, and among the patients who do not have a KIT or PDGFRA mutation, $7-15 \%$ have now been found to harbor a BRAF V600E mutation (28). The patient evaluated in the present case report had a rare PDGRFA A107V mutation in exon 3, with a mutation frequency of $\sim 9.4 \%$, which was considered as an uncommon pathogenic mutation, according to the review of previous studies. The association of this mutation with the occurrence of GIST or imatinib resistance is worthy of further investigation. GISTs usually differ in size, and this is associated with different degrees of risk: Ultra-low risk, $<2 \mathrm{~cm}$; low-risk, $2-5 \mathrm{~cm}$; moderate-risk, 5-10 cm; and high-risk, $>10 \mathrm{~cm}$ (29). Surgical treatment is the first therapeutic choice and is considered as the only possible cure for GISTs (26). High-risk patients have a high rate of postoperative recurrence and metastasis (up to $55-90 \%$ ); $80 \%$ have a 3-4 local recurrence within 1-2 years of surgery and $50 \%$ have liver metastases (30). Imatinib is a KIT/PDGFRA TKI that is mainly used for KIT-positive patients with non-operable, metastatic or recurrent tumors, or as an adjuvant therapy for primary KIT-positive GIST patients (30). In the Scandinavian/German SSG XVIII/AIO trial (31), patients at high risk or with metastasis following $\mathrm{R} 0 / \mathrm{R} 1$ resection were divided into two groups. One group was treated with imatinib (400 mg/day) for 3 years and the other group was treated with imatinib (400 mg/day) for 1 year. The median follow-up time was 4.5 years, and the 5-year PFS times for the two groups were 65 and $48 \%$, respectively. PFS and OS times were longer in the 3-year group. Furthermore, treatment with imatinib for 5 years was found to delay recurrence in $90 \%$ 
of patients, whereas treatment for 3 years only delayed recurrence in $66 \%$ of patients. The 5-year OS time did not notably differ in the 3-year arm of the SSG XVIII trial when compared with the 5-year arm (95 vs. 92\%, respectively). Recurrence of GISTs occurred twice in the patient described in the present case study, following the initial surgery in 1997. As imatinib was listed and included in the medical insurance in China, the patient began to take imatinib (400 mg/day) from May 2013. Regular follow-up with abdominal ultrasound showed no abnormalities. Considering the three recurrences, the patient did not stop the intake of imatinib, and the treatment continued for 5 years.

MFS, formerly known as a myxoid variant of malignant fibrous histiocytoma, is the most frequently occurring sarcoma of the extremities in adults, with characteristic high local recurrence rates (32). At present, the World Health Organization (32) describes MFS as a spectrum of malignant fibroblastic lesions with myxoid stroma, pleomorphism and curvilinear vessels. MFS mainly occurs in the extremities of elderly patients, particularly in the lower limbs; however, it can also appear on the trunk and in the head and neck regions (33). An MFS diagnosis is based on characteristic histopathological features, including the presence of alternating hypocellular myxoid and hypercellular fibrous areas, pleomorphic nuclei, curvilinear thin-walled blood vessels prominent in myxoid areas, the aggregation of neoplastic cells or inflammatory cells, and spindle and stellate cells in the myxoid matrix (34). In previous studies, immunological staining often revealed vimentin and CD34 positivity, which also indicated that the tumor was of fibroblast origin, and the samples could also be SMA-positive and S-100 protein-negative (34). Compared with other soft-tissue sarcoma types, MFS showed better overall prognosis, with an OS rate of $\sim 70 \%$ and an overall risk of metastases between 20 and $25 \%$ in high-grade variants. However, MFS is inclined to have a higher rate of local recurrence compared with other types, with a rate reported to be between 20 and $75 \%$ (33). Previous studies reported that the distant metastasis rate of MFS patients ranged from 9.5-23.6\%, and the lung was considered to be the most common metastatic organ of soft-tissue sarcomas (35). Clinically, once the local recurrence of MFS occurs, the stages and grades of the tumors also increase, leading to metastatic disease (36). Treatment strategies for MFS include extensive local resection, chemotherapy and radiotherapy. The recurrence rate after extensive resection ranged from $16-54 \%$, and it was therefore recommended as the treatment strategy for low-grade MFS (37). In the present study, 2 years after the patient accepted the first surgery, the PET-CT scan showed bone destruction in L5, with a maximum standardized uptake value of $\sim 11.2$, suggesting regional recurrence of fibrous sarcoma. The treatment options were radiotherapy or surgery. In view of the fact that the patient showed no obvious discomfort and possessed a physique unsuitability for radiotherapy or surgery, close observation was suggested.

The patient was treated with three targeted drugs, including imatinib, dabrafenib and trametinib, and to the best of our knowledge, no study has previously assessed the adverse effects of this combination. A previous study demonstrated that the most common adverse reactions to dabrafenib and trametinib were pyrexia, peripheral edema, increased aspartate aminotransferase and blood alkaline phosphatase levels, nasopharyngitis, erythema, stomatitis and headaches (38). Few studies demonstrated that the adverse effects associated with the eye should not be ignored. These effects included retinal vein occlusion, with an incidence of $<1.5 \%$ with trametinib, chorioretinopathy, with an incidence of up to $2 \%$ with both trametinib and dabrafenib, and uveitis, with unknown incidence (39). Toxicities as a result of imatinib treatment include the retention of fluid, nausea, diarrhea, fatigue, abdominal pain, muscle cramps and rashes. Symptoms of congestive heart failure were observed in $\sim 8.2 \%$ of the patients treated with imatinib for GISTs (40). Following the use of imatinib, the patient in the present case had mild edema and diarrhea, which continued for $\sim 6$ months. However, these adverse reactions gradually resolved without any intervention. Since April 2018, combined dabrafenib and trametinib treatment was used, and the patient showed no signs of other significant adverse reactions.

The potential of genetic mutations of ambiguous significance as therapeutic indicators was investigated in the present case report. For example, the patient had a NTRK3 P562L mutation in exon 15. A previous study demonstrated that larotrectinib had marked and durable antitumor activity in patients with tropomyosin receptor kinase fusion-positive cancer, regardless of the age of the patient or of the type of the tumor, and the overall response rate was $75 \%(41)$. The patient in the present case also had a BTK E96K mutation in exon 4, with a mutation frequency of $\sim 20.83 \%$. Ibrutinib was previously demonstrated to significantly improve the prognosis of patients with mantle cell lymphoma who had high expression of BTK (42), and ths drug could be considered as treatment for the present patient. Additionally, PD-L1 positivity (70\%) enables the use of PD1/PD-L1 inhibitors. Thus, the decision on the best treatment option may benefit from the knowledge on the patients genetic mutation profile. Moreover, the best time and whether various anticancer agents should be combined require further investigation.

\section{Acknowledgements}

Not applicable.

\section{Funding}

No funding was received.

\section{Availability of data and materials}

The datasets used and/or analyzed during the current study are available from the corresponding author on reasonable request.

\section{Authors' contributions}

LZ, LF and JW collaborated in data collection, literature review and writing the manuscript. $\mathrm{HC}$ analyzed the data from the patient. ZY, HW and YD were responsible for the treatment and management of the patient. All authors were involved in 
writing the manuscript. All authors read and approved the final manuscript.

\section{Ethics approval and consent to participate}

Not applicable.

\section{Patient consent for publication}

Verbal consent for publication was provided by the patient included in this case report.

\section{Competing interests}

The authors declare that they have no competing interests.

\section{References}

1. DE Luca A, Frusone F, Vergine M, Cocchiara R, LA Torre G, Ballesio L, Monti M and Amabile MI: Breast cancer and multiple primary malignant tumors: Case report and review of the literature. In vivo 33: 1313-1324, 2019.

2. Zhao J, Tan Y, Wu Y, Zhao W, Wu J, Ji M, Shi L, Jiang J and Wu C: A rare case of eight multiple primary malignant neoplasms in female patient: A case report and review of the literature. Oncol Lett 9: 587-590, 2015.

3. Mukaiyama Y, Suzuki M, Morikawa T, Mori Y, Takeshima Y Fujimura T, Fukuhara H, Nakagawa T, Nishimatsu H, Kume H and Homma Y: Multiple primary malignant neoplasms of the glottis, renal pelvis, urinary bladder, oral floor, prostate, and esophagus in a Japanese male patient: A case report. World J Surg Oncol 12, 294, 2014

4. Wang H, Hou J, Zhang G, Zhang M, Li P, Yan X and Ma Z: Clinical characteristics and prognostic analysis of multiple primary malignant neoplasms in patients with lung cancer. Cancer Gene Ther: Jan 31, 2019 (Epub ahead of print).

5. Lee J, Park S, Kim S, Kim J, Ryu J, Park HS, Kim SI and Park BW: Characteristics and survival of breast cancer patients with multiple synchronous or metachronous primary cancers. Yonsei Med J 56: 1213-1220, 2015.

6. Mochizuki T, Teraoka A, Akagawa H, Makabe S, Akihisa T, Sato M, Kataoka H, Mitobe M, Furukawa T, Tsuchiya K and Nitta K: Mutation analyses by next-generation sequencing and multiplex ligation-dependent probe amplification in Japanese autosomal dominant polycystic kidney disease patients. Clin Exp Nephrol 23: 1022-1030, 2019.

7. Kataoka Y, Hirano K, Narabayashi T, Hara S, Fujimoto D, Tanaka T, Ebi N, Tomii K and Yoshioka H: Concordance between the response evaluation criteria in solid tumors version 1.1 and the immune-related response criteria in patients with non-small cell lung cancer treated with nivolumab: A multicenter retrospective cohort study. Cancer Chemother Pharmacol 81: 333-337, 2018.

8. Han Y, Shao N, Xi X and Hao X: Use of microwave ablation in the treatment of patients with multiple primary malignant tumors. Thoracic Cancer 8: 365-371, 2017.

9. Warren S and Gates O: Multiple primary malignant tumors: A survey of the literature and statistical study. Am J Cancer 16 1358-1414, 1932.

10. Etiz D, Metcalfe E and Akcay M: Multiple primary malignant neoplasms: A 10-year experience at a single institution from Turkey. J Cancer Res Ther 13: 16-20, 2017.

11. Motuzyuk I, Sydorchuk O, Kovtun N, Palian Z and Kostiuchenko Y: Analysis of trends and factors in breast multiple primary malignant neoplasms. Breast Cancer (Auckl) 12: 117822341875995,2018

12. Parekh JD, Kukrety S, Thandra A and Valenta C: Multiple primary malignant neoplasms in an elderly patient. Cureus 10: e2384, 2018.

13. Huss S, Pasternack H, Ihle MA, Merkelbach-Bruse S, Heitkötter B, Hartmann W, Trautmann M, Gevensleben H, Büttner R, Schildhaus HU and Wardelmann E: Clinicopathological and molecular features of a large cohort of gastrointestinal stromal tumors (GISTs) and review of the literature: BRAF mutations in KIT/PDGFRA wild-type GISTs are rere events. Hum Pathol 62 : 206-214, 2017.
14. Yang Y, Meng Y, Zhang H, Shen X, Li R, Yu L, Liu B and Wang L: Detection of EGFR and BRAF mutations by competitive allele-specific TaqMan polymerase chain reaction in lung adenocarcinoma. Oncol Lett 15: 3295-3304, 2018.

15. Mitsogianni M, Mitsimponas N, Crespo F, Hartmann KA, Klosterhalfen B, Haase S and Giagounidis A: Concomitant non-small cell lung cancer and hairy cell leukemia in a patient harboring BRAF-V600E mutation in both tissues: A case report. Case Rep Oncol 11: 109-113, 2018.

16. Ding X, Zhang Z, Jiang T, Li X, Zhao C, Su B and Zhou C: Clinicopathologic characteristics and outcomes of Chinese patients with non-small-cell lung cancer and BRAF mutation. Cancer Med 6: 555-562, 2017.

17. Khunger A, Khunger M and Velcheti V: Dabrafenib in combination with trametinib in the treatment of patients with BRAF V600-positive advanced or metastatic non-small cell lung cancer: Clinical evidence and experience. Ther Adv Respir Dis 12: 1753466618767611,2018

18. Mufti M, Ching S, Farjami S, Shahangian S and Sobnosky S: A case series of two patients presenting with pericardial effusion as first manifestation of non-small cell lung cancer with BRAF mutation and expression of PD-L1. World J Oncol 9: 56-61, 2018

19. Weart TC, Miller KD and Simone CB 2nd: Spotlight on dabrafenib/trametinib in the treatment of non-small-cell lung cancer: Place in therapy. Cancer Manag Res 10: 647-652, 2018.

20. Kim TJ, Hong SA, Kim O, Kim SJ, Yang JH, Joung EK, Kang JH and Hong SH: Changes in PD-L1 expression according to tumor infiltrating lymphocytes of acquired EGFR-TKI resistant EGFR-mutant non-small-cell lung cancer. Oncotarget 8: 107630-107639, 2017.

21. Su S, Dong ZY, Xie Z, Yan LX, Li YF, Su J, Liu SY, Yin K, Chen RL, Huang SM, et al: Strong programmed death ligand 1 expression predicts poor response and de novo resistance to EGFR TKIs among non-small cell lung cancer patients with EGFR mutation. J Thorac Oncol 13: 1668-1675, 2018.

22. Jiang X, Zhou J, Giobbie-Hurder A, Wargo J and Hodi FS: The activation of MAPK in melanoma cells resistant to BRAF inhibition promotes PD-L1 expression that is reversible by MEK and PI3K inhibition. Clin Cancer Res 19: 598-609, 2013.

23. Li SD, Martial A, Schrock AB and Liu JJ: Extraordinary clinical benefit to sequential treatment with targeted therapy and immunotherapy of a BRAF V600E and PD-L1 positive metastatic lung adenocarcinoma. Exp Hematol Oncol 6: 29, 2017.

24. Liu J, Chen Z, Chen H, Hou Y, Lu W, He J, Tong H, Zhou Y and Cai W: Genetic polymorphisms contribute to the individual variations of imatinib mesylate plasma levels and adverse reactions in chinese GIST patients. Int J Mol Sci 18: pii E603, 2017.

25. Peixoto A, Costa-Moreira P, Silva M, Santos AL, Lopes S, Vilas-Boas F, Moutinho-Ribeiro P and Macedo G: Gastrointestinal stromal tumors in the imatinib era: 15 years' experience of a tertiary center. J Gastrointest Oncol 9: 358-362, 2018.

26. Sanchez-Hidalgo JM, Duran-Martinez M, Molero-Payan R, Rufian-Peña S, Arjona-Sanchez A, Casado-Adam A, Cosano-Alvarez A and Briceño-Delgado J: Gastrointestinal stromal tumors: A multidisciplinary challenge. World J Gastroenterol 24: 1925-1941, 2018.

27. Rubin BP: Gastrointestinal stromal tumours: An update. Histopathology 48: 83-96, 2006.

28. Balachandran VP and Dematteo RP: Gastrointestinal stromal tumors: Who should get imatinib and for how long. Adv Surg 48: $165-183,2014$.

29. Alecu L, Tulin A, Enciu O, Bărbulescu M, Ursuţ B and Obrocea F: Gastrointestinal stromal tumors-Diagnosis and surgical treatment. Chirurgia (Bucur) 110: 525-529, 2015.

30. Mei L, Du W, Idowu M, von Mehren M and Boikos S: Advances and challenges on management of gastrointestinal stromal tumors. Front Oncol 8: 135, 2018.

31. Joensuu H, Eriksson M, Sundby Hall K, Hartmann JT, Pink D, Schütte J, Ramadori G, Hohenberger P, Duyster J, Al-Batran SE et al: One vs three years of adjuvant imatinib for operable gastrointestinal stromal tumor: A randomized trial. JAMA 307: 1265-1272, 2012.

32. De Vita A, Recine F, Mercatali L, Miserocchi G, Liverani C, Spadazzi C, Casadei R, Bongiovanni A, Pieri F, Riva N, et al: Myxofibrosarcoma primary cultures: Molecular and pharmacological profile. Ther Adv Med Oncol 9: 755-767, 2017.

33. 33, Colia V, Fiore M, Provenzano S, Fumagalli E, Bertulli R, Morosi C, Dei Tos AP, Barisella M, Gronchi A, Casali PG and Sanfilippo R: Activity of anthracycline- and ifosfamide-based chemotherapy in a series of patients affected by advanced myxofibrosarcoma. Clin Sarcoma Res 7: 16, 2017. 
34. Wong A, Chan Woo Park R, Mirani NM and Eloy JA Myxofibrosarcoma of the maxillary sinus. Allergy Rhinol (Providence) 8: e95-e99, 2017.

35. Tsuchie H, Kaya M, Nagasawa H, Emori M, Murahashi Y, Mizushima E, Miyakoshi N, Yamashita T and Shimada Y: Distant metastasis in patients with myxofibrosarcoma. Ups J Med Sci 122: 190-193, 2017.

36. Lohberger B, Stuendl N, Leithner A, Rinner B, Sauer S, Kashofer K and Liegl-Atzwanger B: Establishment of a novel cellular model for myxofibrosarcoma heterogeneity. Sci Rep 7: 44700, 2017.

37. Nomura T, Sakakibara S, Moriwaki A, Kawamoto T, Suzuki S, Ishimura T, Hashikawa K and Terashi H: Terashi, low-grade myxofibrosarcoma of the rectus abdominus muscle infiltrating into abdominal cavity: A case report. Eplasty 17: e6, 2017.

38. Yamazaki N, Tsutsumida A, Takahashi A, Namikawa K Yoshikawa S, Fujiwara Y, Kondo S, Mukaiyama A, Zhang F and Kiyohara Y: Phase 1/2 study assessing the safety and efficacy of dabrafenib and trametinib combination therapy in Japanese patients with BRAF V600 mutation-positive advanced cutaneous melanoma. J Dermatol 45: 397-407, 2018.

39. Dy GK and Adjei AA: Understanding, recognizing, and managing toxicities of targeted anticancer therapies. CA Cancer J Clin 63: 249-279, 2013.
40. Ghias AAP, Bhayani S, Gemmel DJ and Garg SK: Rapidly progressive dyspnea in gastrointestinal stromal tumor (GIST) with imatinib cardiac toxicity. J Community Hosp Intern Med Perspect 8: 87-91, 2018.

41. Drilon A, Laetsch TW, Kummar S, DuBois SG, Lassen UN Demetri GD, Nathenson M, Doebele RC, Farago AF, Pappo AS, et al: Efficacy of larotrectinib in TRK fusion-positive cancers in adults and children. N Engl J Med 378: 731-739, 2018.

42. Noy A, de Vos S, Thieblemont C, Martin P, Flowers CR, Morschhauser F, Collins GP, Ma S, Coleman M, Peles S, et al: Targeting Bruton tyrosine kinase with ibrutinib in relapsed/refractory marginal zone lymphoma. Blood 129: 2224-2232, 2017.

$$
\begin{aligned}
& \text { This work is licensed under a Creative Commons } \\
& \text { Attribution-NonCommercial-NoDerivatives } 4.0 \\
& \text { International (CC BY-NC-ND 4.0) License. }
\end{aligned}
$$

